\title{
Aerodynamic Shape Optimization of Supersonic Wings by Adaptive Range Multiobjective Genetic Algorithms
}

\author{
Daisuke Sasaki ${ }^{1}$, Masashi Morikawa ${ }^{1}$, Shigeru Obayashi $^{2}$ and Kazuhiro Nakahashi ${ }^{3}$ \\ ${ }^{1}$ Tohoku University, Department of Aeronautics and Space Engineering, Nakahashi Lab., \\ 980-8579 Sendai, Japan \\ \{sasaki, morikawa\}ead.mech.tohoku.ac.jp \\ ${ }^{2}$ Tohoku University, Institute of Fluid Science, \\ 980-8577 Sendai, Japan \\ obayashilieee.org \\ ${ }^{3}$ Tohoku University, Department of Aeronautics and Space Engineering, \\ 980-8579 Sendai, Japan \\ nakahashead.mech.tohoku.ac.jp
}

\begin{abstract}
This paper describes an application of Adaptive Range Multiobjective Genetic Algorithms (ARMOGAs) to aerodynamic wing optimization. The objectives are to minimize transonic and supersonic drag coefficients, as well as the bending and twisting moments of the wings for the supersonic airplane. A total of 72 design variables are categorized to describe the wing's planform, thickness distribution, and warp shape. ARMOGAs are an extension of MOGAs with the range adaptation. Four-objective optimization was successfully performed. Pareto solutions are compared with Pareto optimal wings obtained by the previous three-objective optimization and a wing designed by National Aerospace Laboratory (NAL).
\end{abstract}

\section{Introduction}

Evolutionary Algorithms (EAs) have been widely used to solve real-world optimization problems in the various fields with the aid of the rapid progress of the computers and the algorithms themselves. To treat real-world problems, a large search space is often needed. This can lead to slow-down of the convergence and can require a large number of function evaluations. Especially in aerodynamic design, which requires large computational time for performance evaluation, more efficient and effective algorithms are needed. Among several EAs coupled with CFD (Computational Fluid Dynamics) proposed to seek optimal solutions in aerodynamic designs (for example, see [1-3]), Adaptive Range Genetic Algorithms (ARGAs) proposes a unique approach [4].

In the real-world optimization, the multiobjective optimization is often required rather than the single-objective optimization since there exist tradeoffs between various objectives in general. EAs have many attractive advantages to solve the multiobjective problem. Since EAs seek optimal solutions in parallel, multiple Pareto solutions can be obtained simultaneously without specifying weights between objectives [5]. In the aerodynamic multiobjective optimization problem, efficient and 
effective algorithms are required to reduce the large computational time. In this study, Adaptive Range Multiobjective Algorithms (ARMOGAs) developed from ARGAs for multiobjective optimization are applied to the aerodynamic multiobjective design optimization.

Aerodynamic design for supersonic transport (SST) is considered in this study. A next-generation SST is required to improve the supersonic cruising performance and to prevent the sonic boom. However, there is a severe tradeoff between lowering the drag and boom. Therefore, the next-generation SST may cruise at a supersonic speed only over the sea. This means that it is important to improve not only supersonic performance but also transonic performance, and thus the multipoint aerodynamic optimization is needed. In addition to the reduction of both aerodynamic drags, structural constraints should be considered to keep the wings from having impractically large aspect ratios.

Three-objective optimization for supersonic wings, which minimized transonic and supersonic drag coefficients and the bending moment at the wing root, were reported in [6-7]. In order to consider the viscous effect, a Navier-Stokes solver was used to evaluate the aerodynamic performances at both transonic and supersonic conditions [7]. Successful results were obtained by the multiobjective optimization. There were Pareto solutions that outperformed the NAL's second design in all three objectives, and those wings were similar to the "arrow wing" planform. Although the arrow wing is known to be good for supersonic aerodynamics, it is known to have aeroelastic and control problems due to a large sweep angle. The primary concern is a pitching (twist) moment of the wing. The design results also showed that the second derivative of the wing thickness distribution was discontinuous. This lead to another concern of the designer for the possible boundary layer separation at the maximum thickness location. Therefore the minimization of the pitching moment is added as the present fourth objective with an improved wing thickness parameterization.

National Aerospace Laboratory (NAL) in Japan is working on the scaled experimental supersonic airplane project (NEXST-I) [8]. A scaled experimental supersonic airplane without a propulsion system will be launched with a rocket in 2002. The airplane will be separated from the rocket after launch and will glide down to sample the flight data in the supersonic region. The flight data will be compared with the CFD results to validate the reliability and accuracy of CFD predictions. NAL designed several configurations for the experimental aircraft. The present Pareto solutions obtained are compared with the NAL's design. In order to verify the present optimization method, the present Pareto solutions are also compared with the Pareto solutions obtained before.

\section{Adaptive Range Multiobjective Genetic Algorithms}

To reduce the large computational burden, the reduction of the total number of evaluations is needed. On the other hand, a large string length is necessary for real parameter problems. ARGAs, which originally proposed by Arakawa and Hagiwara, are a quite unique approach to solve such problems efficiently [9-10]. Oyama 
developed real-coded ARGAs and applied them to the transonic wing optimization [4].

\subsection{Real-Coded Adaptive Range Genetic Algorithms}

The main difference between ARGAs and conventional GAs is the introduction of the range adaptation. The flowchart of ARGAs is shown in Fig. 1. Population is reinitialized every $\mathrm{M}$ generations for the range adaptation so that the population advances toward promising regions. Another difference is the elimination of the range limits because design variables are encoded into the normal distribution.

In the real-coded ARGAs, the real value of $i$-th design variable $P_{i}$ is encoded to a real number $r_{i}$ defined in $(0,1)$ so that $r_{i}$ is equal to the integrations of the normal distribution form $-\infty$ to $P n_{i}$,

$$
\begin{gathered}
r_{i}=\int_{-\infty}^{\mathrm{pn}_{\mathrm{i}}} N(0,1)(z) d z \\
P_{i}=\sigma_{i} \cdot p n_{i}+\mu_{i}
\end{gathered}
$$

where the average $\mu_{i}$ and the standard deviation $\sigma_{i}$ of $i$-th design variable are calculated by sampling the top half of the previous population to promote the population toward search regions of high fitness. A schematic view of this coding is illustrated in Fig. 2.

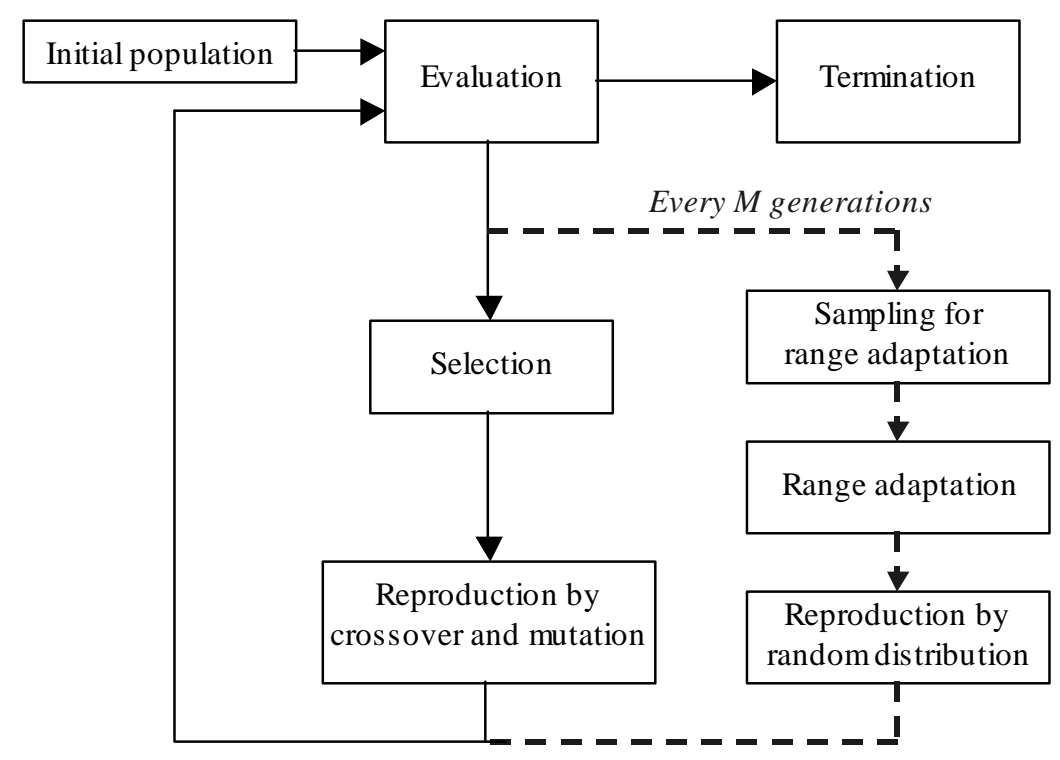

Fig. 1. Flowchart of ARGAs 


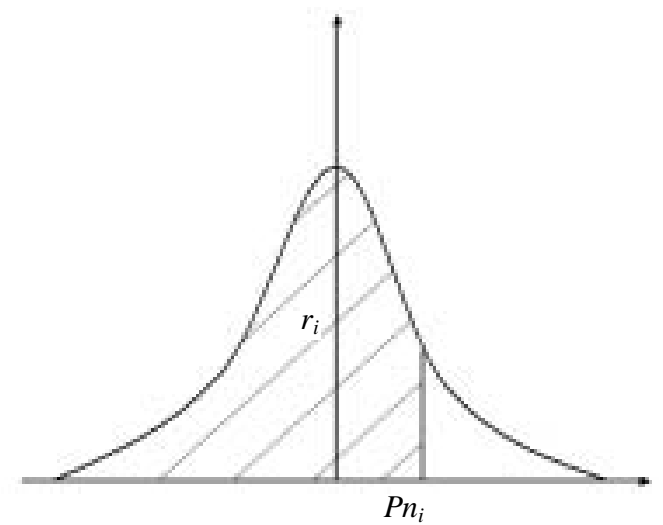

Fig. 2. Normal distribution is used for encoding in ARGAs

\subsection{Extension of ARGAs to the Multiobjective Problem}

In this study, ARGAs have to deal with multiple Pareto solutions for the multiobjective optimization. The basis of ARMOGAs is the same as ARGAs, but a straightforward extension may cause a problem in the diversity of the population. To better preserve the diversity of solution candidates, the normal distribution for encoding is changed as shown in Fig. 3. The searching region is partitioned into three parts (i, ii, iii). The region i and iii make use of the same encoding method as ARGAs. In contrast, the region ii adopts the conventional real-number encoding method.

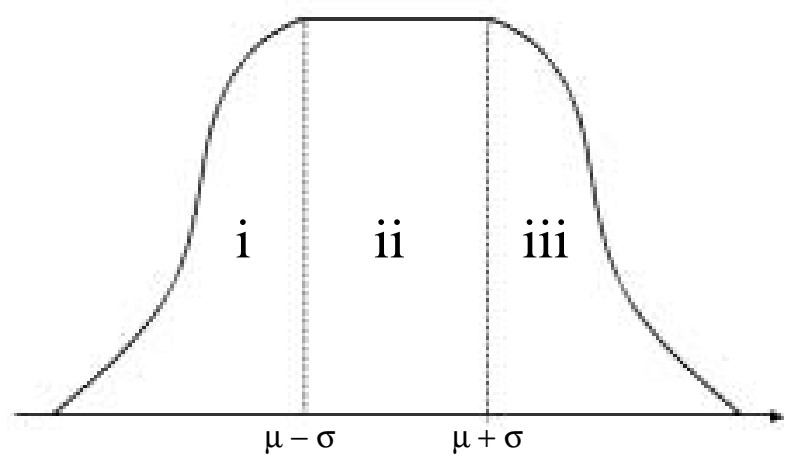

Fig. 3. Normal distribution used in ARGAs' encoding is extended to maintain the diversity of the population for ARMOGAs 


\section{Problem Definitions}

\subsection{Objective Functions}

The objective functions used in this study can be stated as follows:

1. Drag coefficient at transonic cruise, $C_{D, t}$

2. Drag coefficient at supersonic cruise, $\mathrm{C}_{\mathrm{D}, \mathrm{s}}$

3. Bending moment at the wing root at supersonic cruise condition, $\mathbf{M}_{\mathrm{B}}$

4. Twisting moment at supersonic cruise condition, $\mathrm{M}_{\mathrm{T}}$

In the present optimization, all four objective functions are to be minimized. Both the transonic and supersonic drag coefficients are evaluated by a Navier-Stokes solver. Both the bending and twisting moments are calculated by directly integrating the computed pressure load at the supersonic condition. The bending moment represents the lateral moment that acts at the wing root. The twisting moment is the pitching moment measured at the leading edge of the root along the line normal to the root. The present optimization is performed at two design points for the transonic and supersonic cruises. Each flow conditions and the target lift coefficients are described below.

1. Transonic cruising Mach number, $\mathrm{M}_{\infty, \mathrm{t}}=0.9$

2. Supersonic cruising Mach number, $M_{\infty, s}=2.0$

3. Target lift coefficient at transonic cruising condition, $\mathrm{C}_{\mathrm{L}, \mathrm{t}}=0.15$

4. Target lift coefficient at supersonic cruising condition, $\mathrm{C}_{\mathrm{L}, \mathrm{s}}=0.10$

5. Reynolds number based on the root chord length at both conditions, $\operatorname{Re}=1.0 \times 10^{7}$

To maintain constant lift constraints, the angle of attack is predicted by using $\mathrm{C}_{\mathrm{L} \alpha}$ obtained from the finite difference. Thus, three Navier-Stokes computations per evaluation are required.

\subsection{Design Parameters}

Design variables are categorized to planform, warp shape and the thickness distribution. The definitions of design parameters are same as the previous optimization except for the thickness distribution. As mentioned earlier, the previous thickness definition has the lack of smoothness at the maximum thickness as shown in Fig. 4 [11]. To improve it, two more control points, which are symmetrical with respect to maximum thickness location, are added as shown in Fig. 5. Therefore, the present definition makes the second derivative continuous at the maximum thickness. As a result, 11 control points are used to represent the thickness distribution by a Bezier curve at three spanwise sections (root, kink and tip). Linear interpolation is used to interpolate the thickness distribution in spanwise direction. Table 1 describes the constraints for the thickness definition. 
The wing planform is determined by six design variables as shown in Fig. 6. Since the wing area is fixed, the chord length at the wing tip is determined automatically. Constraints and the range of design variables are described in Tab. 2. The warp shape is composed of camber and twist. The camber surface is defined from the airfoil camber lines at the inboard and outboard of the wing separately. Each surface is represented by the Bezier surface which has four polygons in the chordwise direction and three in the spanwise direction. In case of the wing twist, a B-spline curve with six polygons is used. The total number of design parameters becomes 72 .

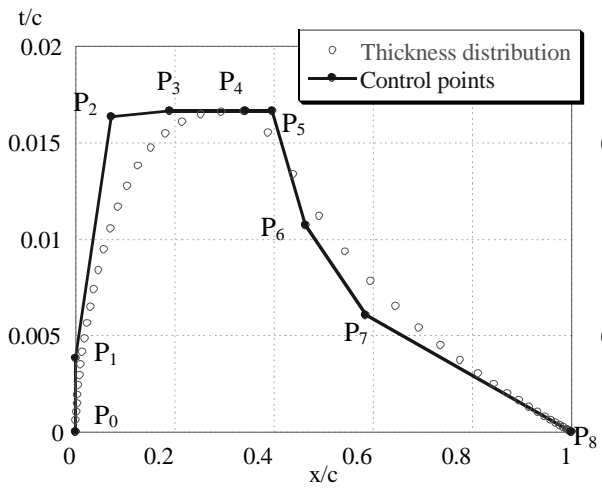

Fig. 4. Previous thickness definition

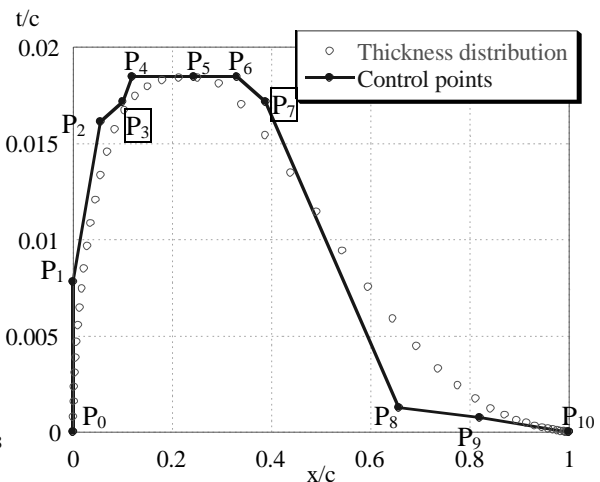

Fig. 5. Present thickness distribution

Table 1. Constraints for thickness distribution

\begin{tabular}{ll}
\hline Maximum thickness & $3<\mathrm{Z}_{\mathrm{P}_{5}}<4$ \\
Maximum thickness location & $15<\mathrm{X}_{\mathrm{P}_{5}}<70$ \\
First derivative constant at $\mathrm{P}_{5}$ & $\mathrm{Z}_{\mathrm{P}_{4}}=\mathrm{Z}_{\mathrm{P}_{5}}=\mathrm{Z}_{\mathrm{P}_{6}}$ \\
Second derivative constant at $\mathrm{P}_{5}$ & $\mathrm{X}_{\mathrm{P}_{5}}-\mathrm{X}_{\mathrm{P}_{3}}=\mathrm{X}_{\mathrm{P}_{7}}-\mathrm{X}_{\mathrm{P}_{5}}, \mathrm{Z}_{\mathrm{P}_{3}}=\mathrm{Z}_{\mathrm{P}_{7}}$ \\
First derivative constant at leading edge & $\mathrm{X}_{\mathrm{P}_{0}}=\mathrm{X}_{\mathrm{P}_{1}}$ \\
\hline
\end{tabular}

Table 2. Constraints for planform shape

\begin{tabular}{ll}
\hline Chord length at root & $10<\mathrm{C}_{\text {root }}<20$ \\
Chord length at kink & $3<\mathrm{C}_{\text {kink }}<15$ \\
Inboard span length & $2<\mathrm{b}_{1}<7$ \\
Outboard span length & $2<\mathrm{b}_{2}<7$ \\
Inboard sweep angle (deg) & $35<\alpha_{1}<70$ \\
Outboard sweep angle (deg) & $35<\alpha_{2}<70$ \\
Wing area & $\mathrm{S}=60$ \\
\hline
\end{tabular}




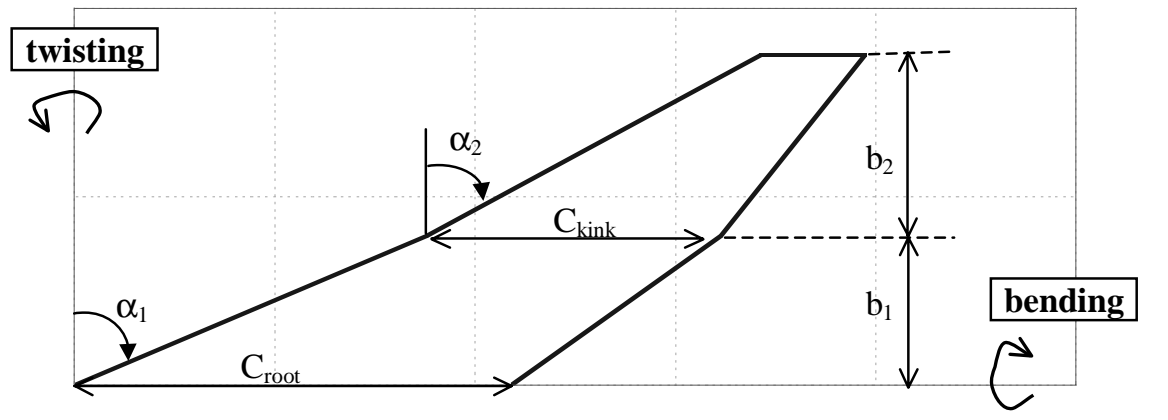

Fig. 6. Wing planform is defined by six design variables. Schematic view of bending and twisting moments are also shown.

\subsection{Evaluation by CFD}

Previous results showed the importance of the viscous effect for wing designs. Thus, the three-dimensional, compressible, thin layer Navier-Stokes code is again used to evaluate aerodynamic performance of a three-dimensional wing at both transonic and supersonic conditions. This Navier-Stokes code employs total-variation-diminishing type upwind differencing and the lower-upper factored symmetric Gauss-Seidel scheme [12]. An algebraic mixing length turbulence model by Baldwin and Lomax is adopted [13]. To accelerate the convergence, the multigrid method is also used [14].

Taking advantage of the characteristic of GAs, the present optimization is parallelized on SGI ORIGIN 2000 at the Institute of Fluid Science, Tohoku University. The system has 640 PE's with peak performance of 384 GFLOPS and 640 GB of memory. The master PE manages the optimization process, while the slave PE's compute the Navier-Stokes code. The population size used in this study was set to 64 so that the process was parallelized with 32-128 PE's depending on the availability, because the transonic and supersonic computations can be processed separately. It should be noted that the parallelization was almost $100 \%$ because almost all the CPU time was dominated by Navier-Stokes computations. The present optimization requires about six hours per each generation parallelized on 128 PEs.

\subsection{Details of the present ARMOGA}

In this study, the design variables are encoded in the real numbers. Blended crossover $(B L X-\alpha)$ is adopted as a crossover operator. This crossover method produces children on a segment defined by two parents and user specified parameter $\alpha$. Parameter $\alpha$ is set to 0.5 except for the planform definition design variables. In the case of the six planform design variables, $\alpha$ is set to 0.0 to prevent the computational divergence of new candidates. After the crossover, mutation takes place at a probability of $20 \%$ based on a uniform random number selected over $10 \%$ of the range. 
Selection is based on the Pareto ranking method and fitness sharing. Each individual is assigned to its rank according to the number of individuals that dominate it. A standard fitness sharing function is used to maintain the diversity of the population. The so-called best- $N$ selection is also employed.

A population is set to 64 , and the range adaptation is performed every 10 generations starting from the $15^{\text {th }}$ generation.

\section{Optimization Results}

\subsection{Overview of Pareto Solutions}

The evolution was computed for 75 generations. After the computation, all the solutions evolved were sorted again to find the final Pareto solutions. The Pareto solutions were obtained in four-dimensional objective function space. To understand the distribution of Pareto solutions, all Pareto solutions are projected into twodimensional objective function space between transonic and supersonic drag coefficients as shown in Fig. 7. In this figure, the Pareto solutions obtained from the previous optimization with three design objectives are also plotted. The present Pareto front is larger than before, in particular, better tradeoff solutions appear in the tradeoff surface I. The planform shapes of the extreme Pareto solutions, which minimize respective objective functions, appear physically reasonable as shown in Fig. 8. A wing, which minimizes the transonic cruising drag, has a less leading-edge sweep and a large aspect ratio. On the contrary, a wing with the lowest supersonic drag coefficient has a large leading-edge sweep to remain inside the Mach cone.

\subsection{Influences of the bending and twisting moments to drag coefficients}

To examine influences of the bending and twisting moments, all the present Pareto solutions in Fig. 7 are labeled by the bending and twisting moments, respectively, as shown in Fig. 9. The wings, which locate near the tradeoff surface between transonic and supersonic drag coefficients (tradeoff surface I, Fig. 7), have impractically large bending moments as shown in Fig.9 (a). The bending moment is closely related to both transonic and supersonic drag coefficients. On the other hand, the twisting moment has an influence only on supersonic drag coefficient. As a consequence, the region II in Fig. 7 was primarily corresponding the minimization of the bending moment, not to the new objective function of the twisting moment minimization. The planform shapes, which have the lowest bending moment obtain/ed by the present and previous optimization respectively, are plotted in Fig. 10. Since these planform shapes are supposed to be indifferent, the present minimum wing and the wings belonged to the region II are found thanks to ARMOGA. Similarly, the improvement of the present tradeoff surface I from the previous result (Fig. 7) is due to ARMOGA.

Pareto solutions are also projected into the two-dimensional plane with the supersonic drag coefficient and the twisting moment in Fig. 11. A clear tradeoff is 
found. Figure 11 is also labeled by aspect ratios but there is no trend in performance based on the aspect ratios.

\subsection{Comparison with NAL's second design and the previous design}

To examine the quality of the present Pareto solutions, two wings are compared with NAL's second design wing as well as the previous wing obtained by three-objective optimization. NAL SST Design Team already finished the fourth aerodynamic design for the scaled experimental supersonic airplane to be launched in 2002 (NEXST-I). To summarize their concepts briefly, the first design determined the planform shapes among 99 candidates, then the second design was performed by the warp optimization based on the linearized theory. The third design aimed a natural-laminar-flow (NLF) wing by an inverse method using a Navier-Stokes code. Finally, the fourth design was performed for a wing-fuselage configuration. Because a fully developed turbulence is assumed in the present Navier-Stokes computations, it is improper to compare the present Pareto solutions to NAL's NLF wing design. Therefore, the NAL second design is chosen for a comparison.

Table 3 summarizes the aerodynamic performances of four wings compared: two present Pareto solutions (A, B), a previous Pareto solution (OBJ-3) and NAL's second design. The aerodynamic calculation of NAL's and the previous design is performed by using the same Navier-Stokes solver. All three Pareto solutions are superior to NAL's second design in all four objectives. The wing planform shapes are compared as shown in Fig. 12. The present and the previous planform shapes are similar to the "arrow wing" type. On the other hand, NAL's planform is similar to the conventional "delta wing" planform. These results indicate that the present arrow wing doesn't necessarily have a large pitching moment because NAL's design has a higher pitching moment.

The thickness distributions at the wing root of three Pareto solutions (A, B, OBJ-3) are presented in Fig. 13. In this figure, Pareto solutions A and B have much smoother thickness distributions than a previous Pareto solution of OBJ-3. The present wings do not have a kink in the thickness distribution thanks to the improved parameterization, and less likely to cause boundary layer separation.

Table 3. Aerodynamic performances of selected four wings

\begin{tabular}{lcccc}
\hline & $\mathrm{C}_{\mathrm{D}, \mathrm{t}}$ & $\mathrm{C}_{\mathrm{D}, \mathrm{s}}$ & $\mathrm{M}_{\mathrm{B}}$ & $\mathrm{M}_{\mathrm{T}}$ \\
\hline Pareto (A) & 0.00998863 & 0.01085439 & 18.15 & 62.35 \\
Pareto (B) & 0.01007195 & 0.01093646 & 17.39 & 60.60 \\
OBJ-3 & 0.01004036 & 0.01093742 & 18.21 & 61.00 \\
NAL2nd & 0.01010175 & 0.01097646 & 18.23 & 63.31 \\
\hline
\end{tabular}




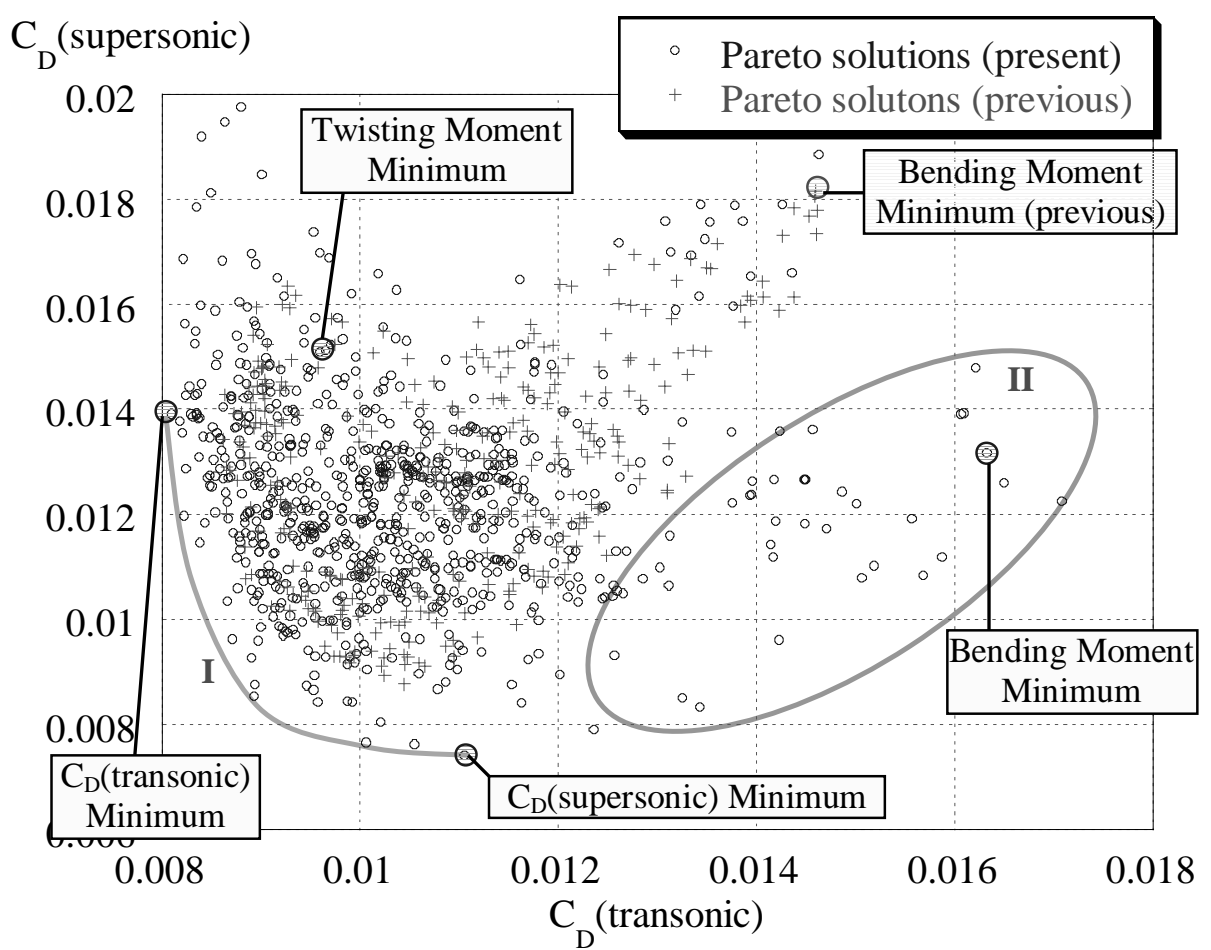

Fig. 7. Projection of present 4-objective Pareto front to transonic and supersonic drag tradeoffs. Pareto solutions obtained by previous 3-objective optimization are also plotted here. Extreme Pareto solutions are indicated. A previous Pareto solutions with the minimum bending moment is also indicated

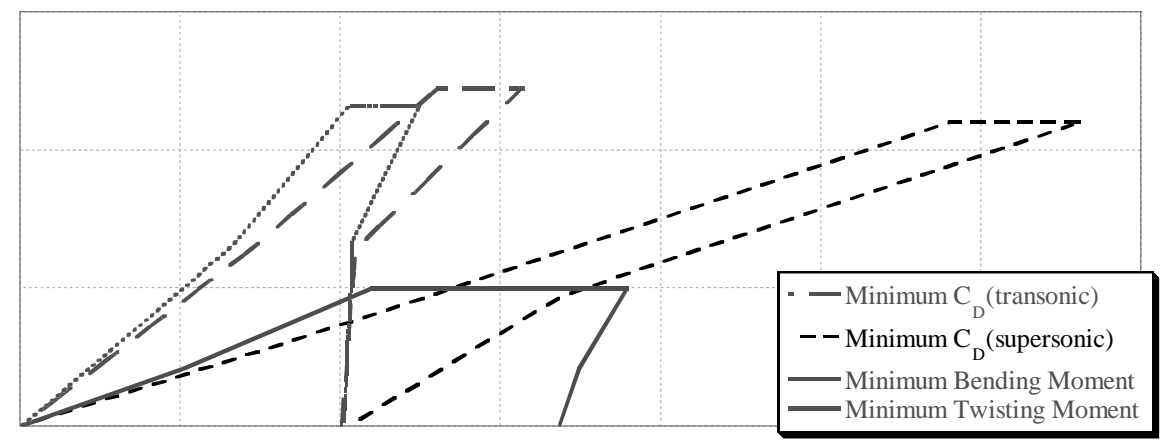

Fig. 8. Typical planform shapes of the extreme Pareto solutions 


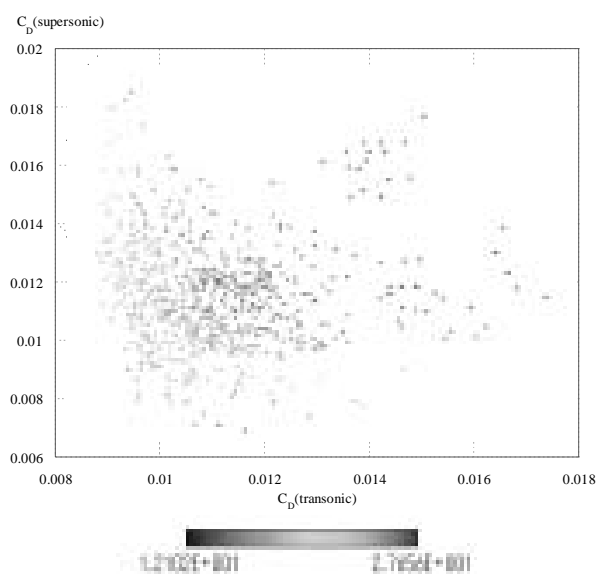

(a) Labeled by bending moment

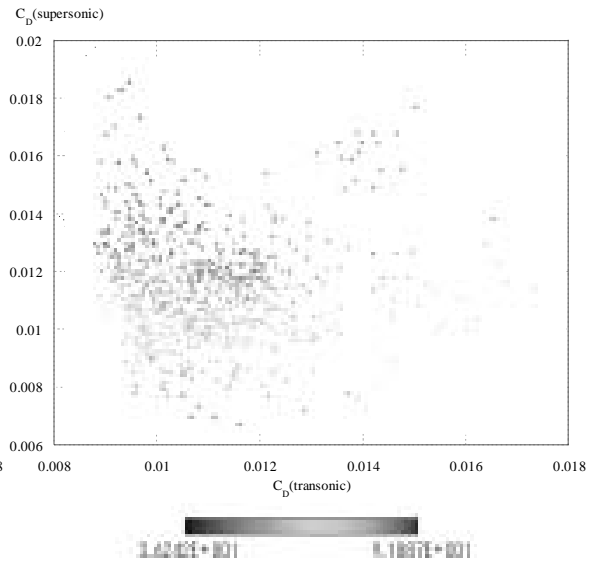

(b) Labeled by twisting moment

Fig. 9. Projection of Pareto front to transonic and supersonic drag tradeoffs labeled by bending and twisting moments

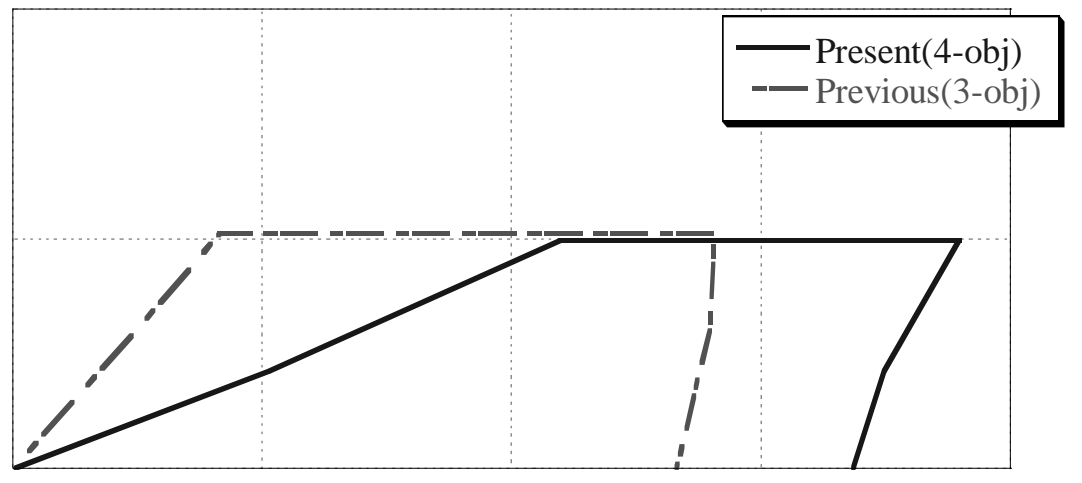

Fig. 10. Comparison of planform shapes having lowest bending moment obtained by the present and previous optimizations 


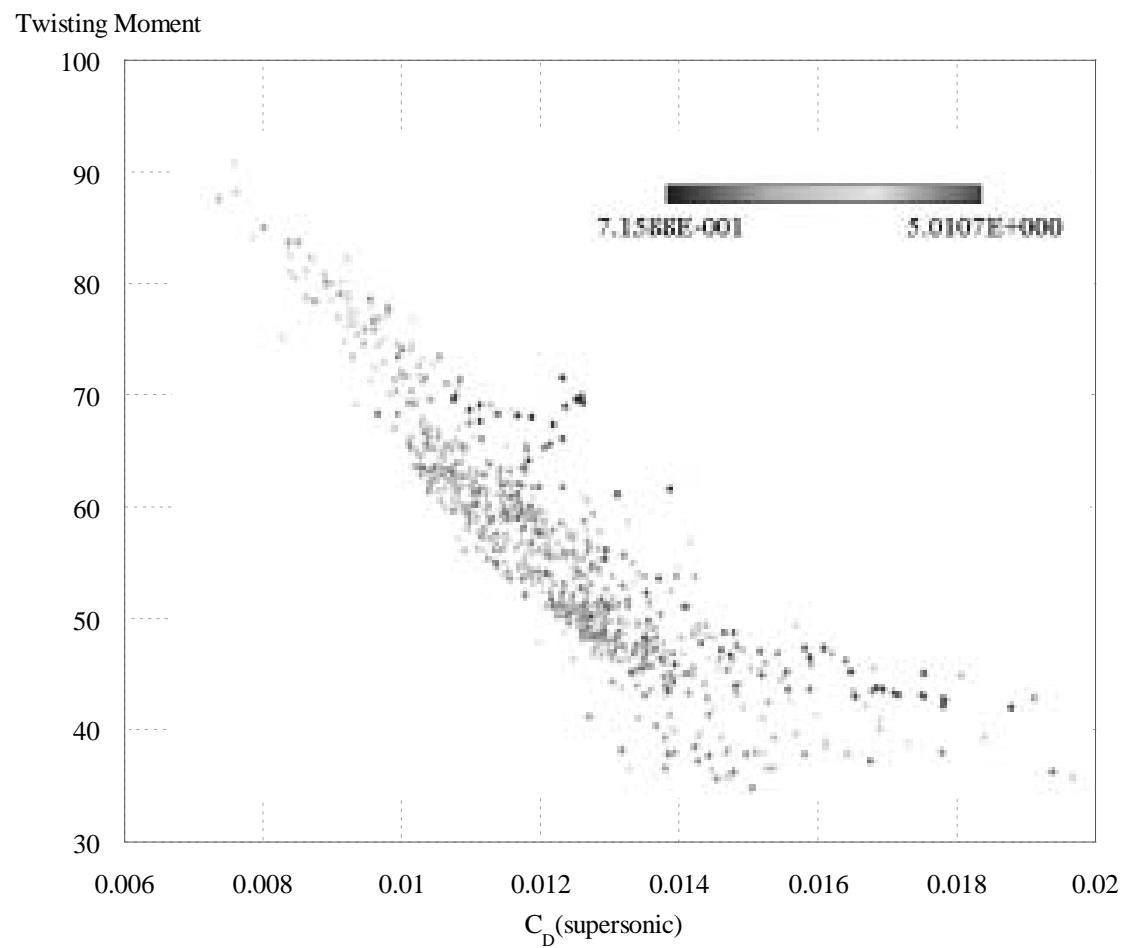

Fig. 11. Projection of Pareto front to supersonic drag and twisting moment tradeoffs labeled by aspect ratios

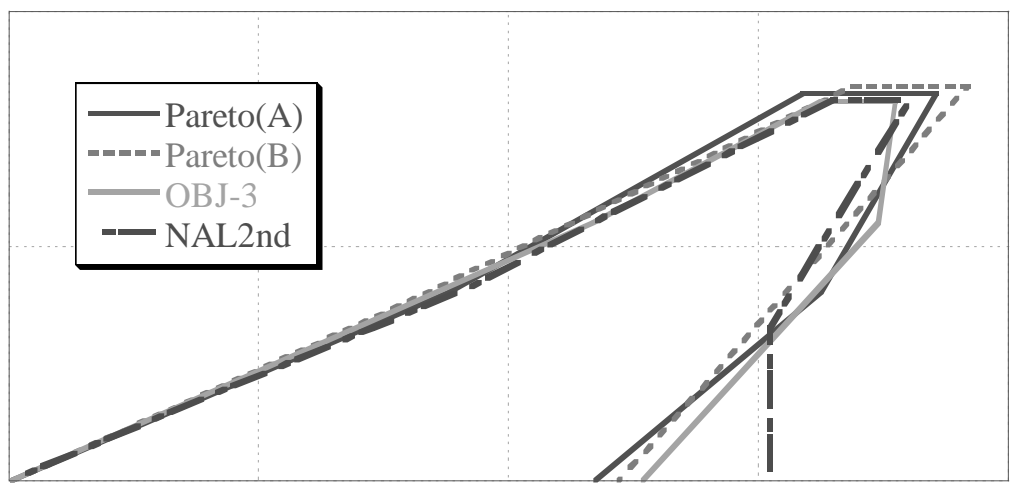

Fig. 12. Comparison of planform shapes among selected Pareto solutions and NAL's design. Planform shapes of the present (A, B) and previous results (OBJ-3) are similar to the "arrow wing" 


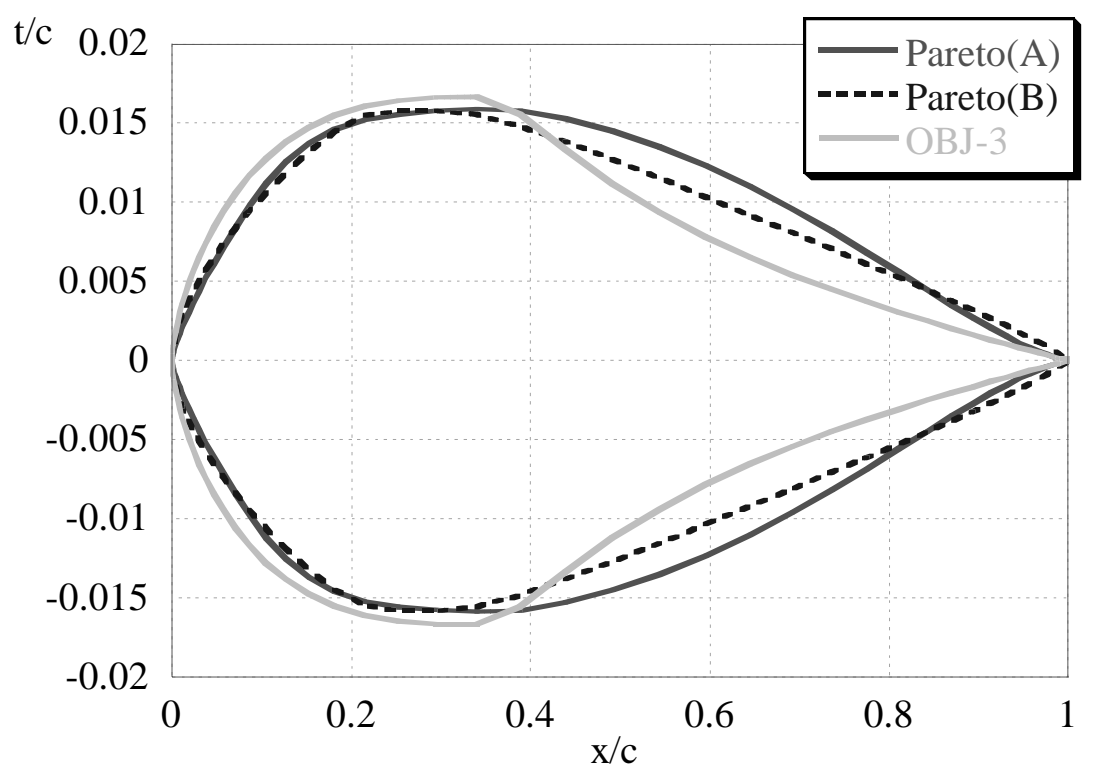

Fig. 13. Comparison of thickness distributions at the wing root among selected Pareto solutions. Thickness distributions of Pareto solutions (A, B) are much smoother at the maximum thickness location than that of the previous result $(\mathrm{OBJ}-3)$ is

\section{Conclusion}

Four-objective aerodynamic optimization of the wings for SST was performed by ARMOGA. In addition to the previous objective functions, which are to minimize the transonic and supersonic drag coefficients and the bending moment at the wing root, the minimization of the twisting moment is added. The number of design variables is increased from 66 to 72 to improve the thickness distribution. A Navier-Stokes solver is used to evaluate the aerodynamic performances.

As a result of the optimization, reasonable Pareto solutions were successfully obtained. The planform configurations of the extreme Pareto solutions are found physically reasonable. The resulting Pareto front appeared better than the previous case thanks to the range adaptation. ARMOGA is confirmed to work well in a large search space. By improving the definition of the thickness distributions, more realistic thickness distributions are obtained.

The present Pareto solutions, which are superior to NAL's second design in all four objective functions, are compared with NAL's wing and an optimal wing obtained before. As for the planform, optimal wings are similar to the "arrow wing" type. On the other hand, the NAL's design is similar to the conventional "delta wing" type. It also shows that even the arrow wing can reduce the pitching moment below that of 
the NAL second design. The present arrow wing is a good design candidate for the next-generation SST.

\section{Acknowledgements}

To perform the wing design for SST, the computation was carried out in parallel using ORIGIN2000 in the Institute of Fluid Science, Tohoku University. This research was partly funded by Japanese Government's Grants-in-Aid for Scientific Research, No. 10305071. The third author's research has been partly supported by Bombardier Aerospace, Toronto, Canada. The authors would like to thank National Aerospace Laboratory's SST Design Team for providing many useful data.

\section{References}

1. Yamamoto, K., Inoue, O.: Applications of Genetic Algorithm to Aerodynamic Shape Optimization. AIAA paper 95-1650 (1995)

2. Quagliarella, D., Periaux, J., Poloni, C., Winter, G. (Eds.): Genetic Algorithms and Evolution Strategies in Engineering and Computer Science. John Wiley and Sons, Chichester (1998)

3. Doorly, D.: Parallel Genetic Algorithms for Optimisation in CFD. In: Winter, G., et al. (Eds.): Genetic Algorithms in Engineering and Computer Science. John Wiley and Sons, Chichester (1995) 251-270

4. Oyama, A., Obayashi, S., Nakamura, S.: Real-Coded Adaptive Range Genetic Algorithm Applied to Transonic Wing Optimization. Lecture notes in Computer Science, Vol. 1917. Springer-Verlag, Berlin Heidelberg New York (2000) 712-721

5. Fonseca, C. M., Fleming, P. J.: Genetic algorithms for multiobjective optimization: formulation, discussion and generalization. Proc. of the $5^{\text {th }}$ Int. Conference on Genetic Algorithms, Morgan Kaufmann Publishers (1993) 416-423

6. Obayashi, S., Sasaki, D., Takeguchi, Y., Hirose, N.: Multiobjective Evolutionary Computation for Supersonic Wing-Shape Optimization. IEEE Transactions on Evolutionary Computation, Vol. 4, No. 2 (2000) 182-187

7. Sasaki, D., Obayashi, S., Sawada, K., Himeno, R.: Multiobjective Aerodynamic Optimization of Supersonic Wings Using Navier-Stokes Equations. Proc. of ECCOMAS 2000 [CD-ROM] (2000)

8. Iwamiya, T.: NAL SST Project and Aerodynamic Design of Experimental Aircraft. Proc. of $4^{\text {th }}$ ECCOMAS Computing Fluid Dynamics Conference, Vol. 2 (1998) 580-585

9. Arakawa, M., Hagiwara, I.: Development of Adaptive Real Range (ARRange) Genetic Algorithms, JSME Int. J., Series C, Vol. 41, No. 4 (1998) 969-977

10. Arakawa, M., Hagiwara, I.: Nonlinear Integer, Discrete and Continuous Optimization Using Adaptive Range Genetic Algorithms, Proc. of 1997 ASME Design Engineering Technical Conferences (1997)

11. Grenon, R.: Numerical Optimization in Aerodynamic Design with Application to a Supersonic Transport Aircraft. Proc. of Int. CFD Workshop for Super-Sonic Transport Design (1998) 83-104

12. Obayashi, S., Grurswamy, G. P.: Convergence Acceleration of a Navier-Stokes Solver for Efficient Static Aeroelastic Computations. AIAA Journal, Vol. 33 (1995) 1134-1141 
13. Baldwin, B. S., Lomax, H.: Thin Layer Approximation and Algebraic Model for Separated Turbulent Flows. AIAA paper 78-257 (1978)

14. Jameson, A., Caughey, D. A.: Effect of Artificial Diffusion Scheme on Multigrid Convergence. AIAA Paper 77-635 (1977) 